\title{
Nonlinear dust-acoustic solitary waves in strongly coupled dusty plasmas
}

\author{
S. E. Cousens, ${ }^{1, *}$ S. Sultana, ${ }^{1, \dagger}$ I. Kourakis, ${ }^{1, \ddagger}$ V. V. Yaroshenko, ${ }^{2}$ F. Verheest,${ }^{3,4}$ and M. A. Hellberg ${ }^{4}$ \\ ${ }^{1}$ Centre for Plasma Physics, Department of Physics and Astronomy, Queen's University Belfast, BT7 1NN Northern Ireland, United Kingdom \\ ${ }^{2}$ Max-Planck-Institut für extraterrestrische Physik, 85741 Garching, Germany \\ ${ }^{3}$ Sterrenkundig Observatorium, Universiteit Gent, Krijgslaan 281, B-9000 Gent, Belgium \\ ${ }^{4}$ School of Chemistry and Physics, University of KwaZulu-Natal, Private Bag X54001, Durban 4000, South Africa
}

(Received 2 July 2012; published 10 December 2012)

\begin{abstract}
Dust-acoustic waves are investigated in a three-component plasma consisting of strongly coupled dust particles and Maxwellian electrons and ions. A fluid model approach is used, with the effects of strong coupling being accounted for by an effective electrostatic "pressure" which is a function of the dust number density and the electrostatic potential. Both linear and weakly nonlinear cases are considered by derivation and analysis of the linear dispersion relation and the Korteweg-de Vries equation, respectively. In contrast to previous studies using this model, this paper presents the results arising from an expansion of the dynamical form of the electrostatic pressure, accounting for the variations in its value in the vicinity of the wave.
\end{abstract}

DOI: 10.1103/PhysRevE.86.066404

PACS number(s): 52.27.Lw, 52.35.Fp, 52.35.Sb, 52.27.Gr

\section{INTRODUCTION}

A dusty plasma is characterized by the presence of massive, charged dust particles in addition to the electron, ion, and neutral components that are found in ordinary plasmas. These dust particles range in size from nanometres to millimeters, are typically billions of times more massive than protons, and can have between one thousand and several hundred thousand elementary charges [1]. The study of dusty plasmas has become an increasingly important area of research in plasma physics; its scope encompasses a wide variety of fields such as astrophysics, semiconductor manufacturing, and fusion reactors. It is interesting to note that the first scientific study performed in the International Space Station was a dusty plasma physics experiment [2], and it is perhaps an indication of its perceived importance within the scientific community.

The presence of a massive, charged dust component in a usual electron-ion plasma can have profound effects on the dynamics of the system. This article theoretically investigates aspects of two such effects; namely the introduction of the dustacoustic wave (DAW) as a new wave mode and the possibility of strong coupling between the dust particles.

The DAW is a very low frequency mode in which the wave is supported by the inertia of the dust particles, with the restoring force being provided by the pressure of both the electrons and ions. The DAW was first theoretically investigated in 1990 by Rao et al. [3] and experimentally observed by Barkan et al. [4], who were able to produce fascinating images and real-time videos of the propagation of DAWs due to their low phase velocity and the large size of the dust particles. In addition to DAWs, there may also be the associated nonlinear structures such as dust-acoustic solitary waves, which arise due to a balance between nonlinear effects and dispersion. Solitons are a particular type of solitary wave which maintain their shape and speed after interactions and have been extensively studied in mathematics and physics due to their stable structure

\footnotetext{
*scousens01@qub.ac.uk

†basharminbu@gmail.com

†IoannisKourakisSci@gmail.com; i.kourakis@qub.ac.uk
}

and also because they arise as solutions to various exactly solvable models including the Korteweg-de Vries (KdV) and the nonlinear Schrödinger (NLS) equations.

In 1986 Ikezi [5] predicted that a dusty plasma can enter the strongly coupled regime due to the high charge and low temperature of the dust. Here, the coupling parameter, $\Gamma \gg 1$, where we have

$$
\Gamma=\frac{Z_{d}^{2} e^{2}}{4 \pi \epsilon_{0} k_{B} T_{d} a_{d}},
$$

with $Z_{d}, T_{d}$, and $a_{d}=n_{d}{ }^{-1 / 3}$ being the charge number, temperature, and mean interparticle distance of the dust particles, respectively. This prediction was soon verified in plasma discharges [6-8], and strongly coupled plasmas have since become a popular research area among plasma physicists.

The large masses of the dust particles can cause complications when studying strongly coupled dusty plasmas on earth-based experiments. This is because gravity can dominate over some of the more subtle interactions, masking some interesting phenomena. A series of experiments aboard the TEXUS 35 rocket flight [9] and the International Space Station [2] were able to study strongly coupled dusty plasmas in both liquid and crystalline forms in an environment with negligible gravitational effects. Among the interesting results obtained from these experiments was a void, a region which contained plasma but no dust particles, that formed in the center of the discharge due to effects of the ion drag force.

There have subsequently been many approaches used to theoretically study strongly coupled DAWs such as the generalized thermodynamical [10], the quasilocalized charge approximation [11], kinetic [12], and hydrodynamical [13] models. Another model which has recently been applied to study DAWs is the fluid approach presented by Gozadinos et al. [14]. Drawing inspiration from previous space experiments $[2,9]$, they developed a numerical model to simulate crystalline dusty plasmas under microgravity conditions. In this paper they formulated an equation of state for this regime given by

$$
P_{\star} \simeq \frac{N_{n n}}{3} \Gamma k_{B} T_{d} n_{d}(1+\kappa) \exp (-\kappa),
$$


where $N_{\text {nn }}$ is the number of nearest neighbors that determine the dusty plasma's structure and $\kappa$ is the lattice parameter, defined as the mean interparticle distance $n_{d}^{-1 / 3}$, divided by the dynamical Debye screening length, $\lambda_{D}$, such that

$$
\kappa=\frac{1}{\sqrt[3]{n_{d}} \lambda_{D}}
$$

with

$$
\lambda_{D}=\sqrt{\frac{\epsilon_{0} k_{B} T_{i} T_{e}}{e^{2}\left(n_{i} T_{e}+n_{e} T_{i}\right)}},
$$

where $T_{s}$ and $n_{s}$ are the temperature and number density of species $s=e, i$, respectively. This model, although originally developed for crystalline plasma structures, has recently been applied as an approximation to the equation of state for strongly coupled plasmas near the liquid-crystal phase transition. This has included the study of Bohm sheaths [15], doublelayer formation [16,17], the linear DAW mode [18], and nonlinear solitary wave structures $[19,20]$. This theory is seen to be in excellent agreement with the experimental observations of linear wave modes, as elegantly demonstrated by Yaroshenko et al., for example, Fig. 5 in Ref. [18]. By considering the form of Eqs. (1) and (2), an effective electrostatic "temperature" was defined such that

$$
k_{B} T_{\star}=\frac{N_{\mathrm{nn}} Z_{d}^{2} e^{2}}{12 \pi \epsilon_{0}} \sqrt[3]{n_{d}}(1+\kappa) \exp (-\kappa),
$$

which is typically a few orders of magnitude larger than the dust kinetic temperature. In doing so, they demonstrated that this model predicts the transition to a thermal mode at high wave numbers for dispersion curves obtained in previous experimental studies [21], which the dust kinetic temperature alone was not great enough to explain.

Our aim here is to investigate linear and nonlinear dustacoustic waves in strongly coupled dusty plasmas. We account for strong coupling between the dust grains by using the model presented by Gozadinos et al. [14], along with the electrostatic temperature approach of Yaroshenko et al. [18]. The reductive perturbation method is employed to derive both the linear dispersion relation and the Korteweg-de Vries equation for this system. We then investigate how the properties of linear and nonlinear waves vary with respect to initial plasma parameters. We choose these parameters to reflect those typically observed in dusty plasma experiments and base them on those presented by Bandyopadhyay et al. [22].

In this paper, the electrons and ions are assumed to follow the Maxwellian distribution, such that their densities are dependent on the local electrostatic potential, which varies with the passing of the wave. The lattice parameter $\kappa$, through its dependence on the dynamically varying screening length $\lambda_{D}$, is dependent on the densities of the Maxwellian species and, thus, also varies with the electrostatic potential. The electrostatic pressure is, thus, a dynamically varying quantity, depending on both $\Phi$, through the $\kappa$ term, and $n_{d}$, both explicitly and through $\kappa$. In this paper, we therefore emphasize that

$$
P_{\star} \equiv P_{\star}\left(n_{d}, \Phi\right)
$$

and so the electrostatic pressure is found to vary due to the perturbations in $n_{d}$ and $\Phi$ in the vicinity of the wave. In previous studies in this area [18,20], an approximation was made such that the electrostatic pressure is only a function of the equilibrium parameters; therefore, an aim of this article is to show what effects this approximation has on the results predicted by this model.

The dust charge number, $Z_{d}$, is a function of the size of the dust particle and of the local plasma conditions. There are various theoretical models which can be used to estimate the charge number, but here we use an orbit motion limited (OML) approach [23], which is based on a balance between electron and ion currents onto the dust particles. In a real physical situation, this quantity should vary dynamically in the vicinity of the wave, but for simplicity in this investigation, we only consider the equilibrium values in determining the charge number of the dust and, therefore, do not apply perturbation theory to $Z_{d}$.

This article is structured as follows. In Sec. II, the fluid equations to model dust-acoustic waves in a strongly coupled plasma are presented, using the equation of state derived by Gozadinos et al. [14]. This is followed in Sec. III by an outline of the reductive perturbation method and an introduction to the concept of "electrostatic temperature perturbations" which are used in this paper. In Sec. IV, the relevant equations used in this study, namely the linear dispersion relation and the $\mathrm{KdV}$ equation, are derived from the normalized fluid model. In Sec. V, a parametric investigation is presented for both the linear and solitary wave cases for typical plasma conditions. In Sec. VI we discuss the effects of strong coupling and of the electrostatic temperature perturbations on the dynamics of the waves. A summary of the most important results and conclusions of this paper is presented in Sec. VII.

\section{MODEL EQUATIONS}

\section{A. System description}

We consider linear and nonlinear acoustic waves propagating through an unbounded, three-component plasma consisting of dust particles, ions, and electrons. The radius, $r_{d}$, and the mass, $m_{d}$, of the dust particles are assumed to be constant. The charge of the dust particles, $q_{d}=Z_{d} e$, is assumed to be constant but with a value determined by the equilibrium plasma parameters. Two forces acting on the dust particles are considered. The first is the electrostatic force which arises from the internally generated electric field of the wave, while the second is from the dust particles electrostatically repelling each other. No gravitational or externally applied electromagnetic fields are applied to the system. In the linear case, the wave propagates due to the inertia of the dust particles being balanced by the restoring effects of the electron and ion pressures. For nonlinear wave propagation, we consider a solitary wave, which is supported due to a balance between nonlinear effects and dispersion. Far from the disturbing effects of the wave, the plasma is considered to be in an equilibrium state. Here the plasma is macroscopically homogeneous, quasineutral, and at rest. In the proximity of the wave, the charged dust particles are perturbed by its generated electrostatic potential. The ions and electrons, which have masses much less than that of the dust particles, are assumed to instantaneously 
redistribute themselves according to the Maxwellian distribution.

\section{B. Fluid equations}

We use the fluid approach to model linear and weakly nonlinear dust-acoustic waves in a 1D strongly coupled plasma in a liquid rather than crystalline state. It would be reasonable to ask whether the fluid equations, typically used for weakly coupled and diffuse plasmas, are applicable in the strongly coupled case. In 1996, however, Wang and Bhattacharjee [24] developed a kinetic theory, including strong coupling from the Klimontovich equation and the Bogolyubov-Born-GreenKirkwood-Yvon (BBGKY) hierarchy, and showed that the Vlasov equation, from which the fluid model may be derived, is still valid in the range $1 \ll \Gamma \ll \Gamma_{\text {cr }}$, where $\Gamma_{\text {cr }}$ is the critical coupling parameter at which crystallization occurs.

The number density of the dust particles obeys the continuity equation such that

$$
\frac{\partial n_{d}}{\partial t}+\frac{\partial}{\partial x}\left(n_{d} u_{d}\right)=0,
$$

where $n_{d}$ and $u_{d}$ are the dust number density and the dust fluid velocity, respectively.

The force arising due to strong coupling between the dust particles is here modelled by an effective electrostatic pressure gradient, where we have the pressure, $P_{\star}=n_{d} k_{B} T_{\star}$, with $T_{\star}$ defined in Eq. (5). It was shown in Ref. [16] that the electrostatic temperature $T_{\star}$ is always a few orders of magnitude higher than the kinetic temperature $T_{d}$ of the dust in the region of the crystal-liquid phase transition. In this paper, we therefore follow this assumption and so neglect the effects of $T_{d}$ on the dynamics of the system. The momentum equation for this system therefore can be written as

$$
m_{d} n_{d}\left(\frac{\partial u_{d}}{\partial t}+u_{d} \frac{\partial u_{d}}{\partial x}\right)=n_{d} Z_{d} e \frac{\partial \Phi}{\partial x}-\frac{\partial P_{\star}}{\partial x} .
$$

The system is then closed by Poisson's equation given by

$$
\frac{\partial^{2} \Phi}{\partial x^{2}}=-\frac{e}{\epsilon_{0}}\left(n_{i}-n_{e}-Z_{d} n_{d}\right),
$$

where we have set the ion charge number, $Z_{i}=1$, for simplicity. The density of the electrons and ions in the proximity of the wave is dependent on their temperature and on the electrostatic potential at that point such that

$$
n_{e}=n_{e 0} \exp \left(\frac{e \Phi}{k_{B} T_{e}}\right)
$$

and

$$
n_{i}=n_{i 0} \exp \left(-\frac{e \Phi}{k_{B} T_{i}}\right) .
$$

Far from the effects of the wave, at equilibrium, Poisson's equation gives the quasineutrality condition such that $n_{i 0}-$ $n_{e 0}-Z_{d} n_{d 0}=0$.

\section{Normalization}

\begin{tabular}{|c|c|}
\hline Parameter & Characteristic scale \\
\hline Temperature & $T_{0}=\frac{Z_{d}^{2} n_{d 0} T_{i} T_{e}}{n_{i 0} T_{e}+n_{e 0} T_{i}}$ \\
\hline Length & $\lambda_{D 0}=\sqrt{\frac{\epsilon_{0} k_{B} T_{0}}{n_{d 0} Z_{d}^{2} e^{2}}}$ \\
\hline Time & $\omega_{p d}^{-1}=\sqrt{\frac{\epsilon_{0} m_{d}}{n_{d 0} Z_{d}^{2} e^{2}}}$ \\
\hline Velocity & $v_{0}=\sqrt{\frac{k_{B} T_{0}}{m_{d}}}$ \\
\hline Number density & $n_{0}=n_{d 0}$ \\
\hline Electrostatic potential & $\Phi_{0}=\frac{k_{B} T_{0}}{Z_{d} e}$ \\
\hline
\end{tabular}

We now normalize the various quantities in Eqs. (7)-(9) by the scalings presented in Table I to obtain a dimensionless system of equations. The variables $n_{d}, u_{d}, \Phi$, and $T_{\star}$ are written
TABLE I. Characteristic scales appearing in this investigation.

in dimensionless form as $n, u, \phi$, and $d$, respectively. The normalized space and time quantities are denoted by a tilde. Equations (7)-(9) thus become

$$
\begin{gathered}
\frac{\partial n}{\partial \tilde{t}}+\frac{\partial}{\partial \tilde{x}}(n u)=0 \\
n\left(\frac{\partial u}{\partial \tilde{t}}+u \frac{\partial u}{\partial \tilde{x}}\right)=n \frac{\partial \phi}{\partial \tilde{x}}-\frac{\partial(n d)}{\partial \tilde{x}},
\end{gathered}
$$

and

$$
\frac{\partial^{2} \phi}{\partial \tilde{x}^{2}} \approx(n-1)+c_{1} \phi+c_{2} \phi^{2}+c_{3} \phi^{3},
$$

where for Poisson's equation the relation $\exp (m \phi) \approx 1+$ $m \phi+(m \phi)^{2} / 2+\cdots$ has been used. The coefficients in Poisson's equation are calculated to be

$$
\begin{gathered}
c_{1}=1, \\
c_{2}=-\frac{(1-\mu)\left(1-\mu \theta^{2}\right)}{2(1+\mu \theta)^{2}}, \\
c_{3}=\frac{(1-\mu)^{2}\left(1+\mu \theta^{3}\right)}{6(1+\mu \theta)^{3}},
\end{gathered}
$$

and, in general,

$$
c_{j}=\frac{(1-\mu)^{j-1}\left[(-1)^{j-1}+\mu \theta^{j}\right]}{j !(1+\mu \theta)^{j}},
$$

where we have

$$
\mu=\frac{n_{e 0}}{n_{i 0}}, \quad \theta=\frac{T_{i}}{T_{e}} .
$$

\section{Grain charging}

We consider the dust particles to have a charge number, $Z_{d}$ which depends on the equilibrium conditions of the plasma, and is constant in time throughout the system. We determine the value of $Z_{d}$ by using an orbit motion limited approach [23] and assuming that the electron and ion currents onto the dust particles are such that $I_{e}+I_{i}=0$. In doing so, we obtain the following equation for $Z_{d}$

$$
Z_{d}=\frac{4 \pi \epsilon_{0} r_{d} k_{B} T_{e}}{e^{2}}\left[W\left(\mu \sqrt{\frac{\theta}{\sigma}} \exp (\theta)\right)-\theta\right],
$$

where $\sigma=m_{e} / m_{i}$ and $W$ denotes the Lambert $W$ function, which has recently been applied to the problem of dust charging [25]. This function is included in programs such as MATLAB and Mathematica and therefore allows a closed 
form representation of the dust charge number, without having to rely on numerical methods. For a derivation of the above equation, see Appendix A.

Equation (20) gives an expression for the charge number at equilibrium in the plasma. However, as the wave passes, the local electron and ion densities will change, resulting in a dynamic variation in charge number. This effect is neglected in this article for simplicity and to facilitate the focus on the variations in the electrostatic temperature in the vicinity of the wave.

\section{REDUCTIVE PERTURBATION METHOD}

The reductive perturbation method involves the expansion, in terms of a small parameter $\epsilon$, of the dynamical variables of the system about their equilibrium states such that

$$
A=A_{0}+\sum_{j=1}^{\infty} \epsilon^{j} A_{j},
$$

where $A$ is the variable to be expanded. Here, the normalized quantities to be expanded are the dust number density $n$, the dust fluid velocity $u$, and the electrostatic potential $\phi$ such that

$$
\begin{gathered}
n=1+\epsilon n_{1}+\epsilon^{2} n_{2}+\cdots, \\
u=\epsilon u_{1}+\epsilon^{2} u_{2}+\cdots
\end{gathered}
$$

and

$$
\phi=\epsilon \phi_{1}+\epsilon^{2} \phi_{2}+\cdots .
$$

From Eq. (5) it is seen that $T_{\star}$ is a function of $n_{d}$ and $\Phi$ through the $\kappa$ term and, thus, varies dynamically. For analytical convenience, we introduce the concept of electrostatic temperature perturbations. For details of the derivation of the following quantities, see Appendix B. By expanding the dynamic variables in Eq. (5), we acquire a form of the normalized electrostatic temperature, $d$, such that

$$
d=d_{0}+\epsilon d_{1}+\epsilon^{2} d_{2}+\cdots,
$$

where the normalized equilibrium electrostatic dust temperature, $d_{0}$, is expressed as

$$
d_{0}=T_{\star 0} / T_{0}
$$

with

$$
k_{B} T_{\star 0}=\frac{N_{\mathrm{nn}} Z_{d}^{2} e^{2}}{12 \pi \epsilon_{0}} \sqrt[3]{n_{d 0}}\left(1+\kappa_{0}\right) \exp \left(-\kappa_{0}\right),
$$

where $\kappa_{0}=1 / \sqrt[3]{n_{d 0}} \lambda \lambda_{D 0}$. The perturbations $d_{1}$ and $d_{2}$ are found to be

$$
d_{1}=d_{11} n_{1}+d_{12} \phi_{1}
$$

and

$$
d_{2}=d_{21} n_{2}+d_{22} \phi_{2}+d_{23} n_{1}^{2}+d_{24} n_{1} \phi_{1}+d_{25} \phi_{1}^{2},
$$

where we have

$$
\begin{gathered}
d_{11}=d_{21}=\frac{d_{0}}{3} \frac{1+\kappa_{0}+\kappa_{0}^{2}}{1+\kappa_{0}}, \\
d_{12}=d_{22}=-d_{0} c_{2} \frac{\kappa_{0}^{2}}{1+\kappa_{0}},
\end{gathered}
$$

$$
\begin{gathered}
d_{23}=\frac{d_{0}}{18} \frac{\kappa_{0}^{3}-3 \kappa_{0}^{2}-2 \kappa_{0}-2}{1+\kappa_{0}}, \\
d_{24}=-\frac{d_{0}}{3} c_{2} \frac{\kappa_{0}^{2}\left(\kappa_{0}-1\right)}{\left(1+\kappa_{0}\right)},
\end{gathered}
$$

and

$$
d_{25}=-\frac{d_{0}}{2}\left(3 c_{3}-c_{2}^{2} \kappa_{0}\right) \frac{\kappa_{0}^{2}}{1+\kappa_{0}} .
$$

\section{DERIVATION OF MODEL EQUATIONS}

\section{A. Linear dispersion relation}

To obtain the linear dispersion relation for this system, the dependent variables in Eqs. (12)-(14) are expanded in a power series of $\epsilon$, as described in Sec. III, with terms higher than $\epsilon^{1}$ neglected. By then assuming oscillatory solutions to the perturbed quantities we obtain the following system of equations:

$$
\begin{gathered}
-\tilde{\omega} n_{1}+\tilde{k} u_{1}=0, \\
-\beta \tilde{k} n_{1}+\tilde{\omega} u_{1}+\alpha \tilde{k} \phi_{1}=0,
\end{gathered}
$$

and

$$
n_{1}+\phi_{1}\left(1+\tilde{k}^{2}\right)=0,
$$

in which $\tilde{\omega}$ and $\tilde{k}$ are the normalized frequency and wave number, respectively, and we have

$$
\alpha=1-d_{12}, \quad \beta=d_{0}+d_{11} .
$$

By combining the above system of equations, it can be seen that we arrive at a dispersion relation

$$
\tilde{\omega}^{2}=\frac{\alpha \tilde{k}^{2}}{1+\tilde{k}^{2}}+\beta \tilde{k}^{2} .
$$

In the long-wavelength limit, Eq. (39) gives the normalized phase velocity $v$ such that

$$
v=\frac{\tilde{\omega}}{\tilde{k}}=\sqrt{1+d_{0}+d_{11}-d_{12}} .
$$

\section{B. KdV equation}

To obtain the $\mathrm{KdV}$ equation, in which we balance nonlinearity with dispersion, we first stretch the space and time coordinates in Eqs. (12)-(14) in the style of Washimi and Taniuti [26] such that

$$
\tilde{\xi}=\epsilon^{1 / 2}(\tilde{x}-v \tilde{t}), \quad \tilde{\tau}=\epsilon^{3 / 2} \tilde{t} .
$$

By then applying the reductive perturbation method as outlined in Sec. III to lowest order in $\epsilon$ we obtain

and

$$
-v \frac{\partial n_{1}}{\partial \tilde{\xi}}+\frac{\partial u_{1}}{\partial \tilde{\xi}}=0
$$

$$
\beta \frac{\partial n_{1}}{\partial \tilde{\xi}}-v \frac{\partial u_{1}}{\partial \tilde{\xi}}-\alpha \frac{\partial \phi_{1}}{\partial \tilde{\xi}}=0,
$$

$$
\frac{\partial n_{1}}{\partial \tilde{\xi}}+\frac{\partial \phi_{1}}{\partial \tilde{\xi}}=0
$$


By integrating Eqs. (42)-(44), noting that the perturbations tend to zero at $\xi \rightarrow \pm \infty$, we obtain equations for the dust fluid density and dust fluid velocity perturbations in terms of the electrostatic potential perturbation such that

$$
n_{1}=-\phi_{1}, \quad u_{1}=-v \phi_{1},
$$

as well as an expression for the normalized phase velocity which is the same as Eq. (40).

To next lowest order in $\epsilon$, we obtain

$$
\begin{gathered}
-\frac{\partial \phi_{1}}{\partial \tilde{\tau}}+2 v \phi_{1} \frac{\partial \phi_{1}}{\partial \tilde{\xi}}-v \frac{\partial n_{2}}{\partial \tilde{\xi}}+\frac{\partial u_{2}}{\partial \tilde{\xi}}=0 \\
-v \frac{\partial \phi_{1}}{\partial \tilde{\tau}}+2\left(\frac{1}{2}+d_{11}-d_{12}+d_{23}-d_{24}+d_{25}\right) \phi_{1} \frac{\partial \phi_{1}}{\partial \tilde{\xi}} \\
+\left(d_{0}+d_{21}\right) \frac{\partial n_{2}}{\partial \tilde{\xi}}-v \frac{\partial u_{2}}{\partial \tilde{\xi}}+\left(d_{22}-1\right) \frac{\partial \phi_{2}}{\partial \tilde{\xi}}=0
\end{gathered}
$$

and

$$
\frac{\partial^{2} \phi_{1}}{\partial \tilde{\xi}^{2}}-n_{2}-\phi_{2}-c_{2} \phi_{1}^{2}=0,
$$

where the relations in Eq. (45) have been used to express the first-order perturbed quantities solely in terms of $\phi_{1}$. By differentiating Eq. (48) with respect to $\tilde{\xi}$, an equation for $\partial n_{2} / \partial \tilde{\xi}$ can be obtained, which then can be substituted into Eq. (47) to get an equation for $\partial u_{2} / \partial \xi$. These expressions for both $\partial n_{2} / \partial \tilde{\xi}$ and $\partial u_{2} / \partial \tilde{\xi}$ can then be substituted into Eq. (46) to obtain an equation of the form

$$
\frac{\partial \phi_{1}}{\partial \tilde{\tau}}+\tilde{A} \phi_{1} \frac{\partial \phi_{1}}{\partial \tilde{\xi}}+\tilde{B} \frac{\partial^{3} \phi_{1}}{\partial \tilde{\xi}^{3}}=0,
$$

in which we have

$$
\begin{gathered}
\tilde{A}=-\frac{\left(1+2 v^{2}+2 \alpha c_{2}+2 \gamma\right)}{2 v}, \\
\tilde{B}=\frac{\alpha}{2 v},
\end{gathered}
$$

where $\alpha$ is defined in Eq. (38) and $\gamma$ is defined as

$$
\gamma=d_{11}-d_{12}+d_{23}-d_{24}+d_{25} \text {. }
$$

Equation (49) can be solved by separation of variables, giving a solution of

$$
\phi_{1}(\tilde{\xi}, \tilde{\tau})=\frac{3 \tilde{U}}{\tilde{A}} \operatorname{sech}^{2}\left[\frac{\tilde{\xi}-\tilde{U} \tilde{\tau}}{\sqrt{\frac{4 \tilde{B}}{\tilde{U}}}}\right],
$$

where $\tilde{U}$ is the normalized velocity of the nonlinear wave in the moving reference frame. We now transform back to the laboratory frame, in which the solitary wave is traveling with a normalized velocity $\tilde{V}$, which is greater than the sound speed in the plasma by an amount $\delta \tilde{V}$. Taking $\phi$ to the first order of $\epsilon$, we then have

$$
\phi(\tilde{x}, \tilde{t})=\phi_{m} \operatorname{sech}^{2}\left[\frac{\tilde{\chi}}{\tilde{\Delta}}\right],
$$

where

$$
\left|\phi_{m}\right|=\frac{3 \delta \tilde{V}}{|\tilde{A}|}, \quad \tilde{\Delta}=\sqrt{\frac{4 \tilde{B}}{\delta \tilde{V}}}
$$

are the amplitude and width of the solitary wave and $\tilde{\chi}=$ $\tilde{x}-\tilde{V} \tilde{t}$.

\section{PARAMETRIC INVESTIGATION}

In this section, we present a parametric investigation based on the equations derived in Sec. IV. In the previous sections, we have demonstrated that both the linear dispersion relation and the $\mathrm{KdV}$ equation are dependent on the following equilibrium quantities: $n_{i 0}, n_{e 0}, T_{i}, T_{e}, \sigma=m_{e} / m_{i}, r_{d}$, and $m_{d}$. To determine the coupling parameter of the system, so its phase state may be estimated, the dust temperature $T_{d}$ must be specified. When considering solitary wave solutions to the $\mathrm{KdV}$ equation, we must also specify the velocity of the solitary wave relative to the sound speed in the plasma, which in dimensional form may be expressed as $\delta V=V-v_{\mathrm{ph}}$. From these nine quantities, the various attributes of the linear and nonlinear wave structures can be derived.

Here, we choose to investigate the effects of varying the densities and temperatures of the Maxwellian species and fix the other quantities. For the purposes of this section, the scalings shown in Table I are inappropriate, since they are dependent on these parameters, so in this section we analyze the dimensional form of the equations. Some of the normalization quantities presented in Table I arise naturally in the algebra, however, so the notation of the various dimensionless quantities will be retained in this section.

We will be using the parameters shown in Table II for this investigation, which are based on those measured by Bandyopadhyay et al. [22]. The value of the ratio $\sigma=m_{e} / m_{i}$ is for the commonly used argon plasma, but here we set the ion charge number, $Z_{i}=1$, for simplicity. We choose $N_{\mathrm{nn}}=12$, corresponding to an fcc crystal lattice. We choose the range of temperatures and densities under investigation such that we expect the dust particles to be strongly coupled, but the plasma is in a fluid rather than crystalline state.

Vaulina and Khrapak [27] found that the coupling parameter required for crystallisation is

$$
\Gamma_{\mathrm{cr}}=\frac{106 \exp \left(\kappa_{0}\right)}{\left(1+\kappa_{0}+\kappa_{0}^{2}\right)},
$$

and, therefore, the equation of state, Eq. (2) is valid in the region in which we have approximately $1<\Gamma<\Gamma_{\text {cr }}$, so we restrict our parameters to values such that this inequality holds. Here we have used the equilibrium quantities to determine the coupling parameter, and therefore we do not take into account the effects of the wave perturbations on the phase state of the system.

TABLE II. Parameters studied in this investigation.

\begin{tabular}{lr}
\hline \hline Parameter & Parameter range \\
\hline$n_{i 0}$ & $(5-8) \times 10^{13} \mathrm{~m}^{-3}$ \\
$n_{e 0}$ & $(1-4) \times 10^{13} \mathrm{~m}^{-3}$ \\
$k_{B} T_{i}$ & $(0.2-1) \mathrm{eV}$ \\
$k_{B} T_{e}$ & $(3-8) \mathrm{eV}$ \\
$k_{B} T_{d}$ & $0.3 \mathrm{eV}$ \\
$r_{d}$ & $0.2 \mu \mathrm{m}$ \\
$m_{d}$ & $1 \times 10^{-13} \mathrm{~kg}$ \\
$\sigma=m_{e} / m_{i}$ & $1.3732 \times 10^{-5}$ \\
$\delta V$ & $2 \mathrm{~mm} / \mathrm{s}$ \\
\hline \hline
\end{tabular}




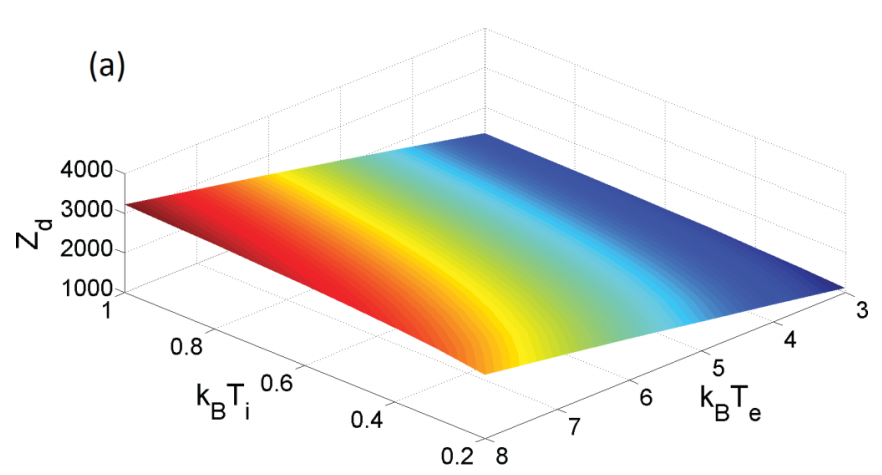

(b)

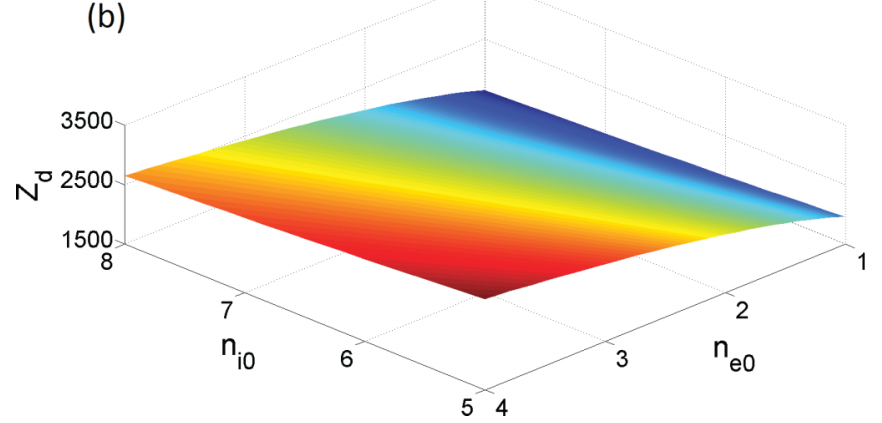

FIG. 1. (Color online) The charge number of the dust particles studied in this investigation, as functions of (a) the temperatures (eV) and (b) the densities (in units of $10^{13} \mathrm{~m}^{-3}$ ) of the Maxwellian species. In both plots we have $m_{d}=1 \times 10^{-13} \mathrm{~kg}, r_{d}=0.2 \mu \mathrm{m}, k_{B} T_{d}=$ $0.3 \mathrm{eV}$, and $\sigma=1.3732 \times 10^{-5}$. In (a) we have $n_{i 0}=7 \times 10^{13} \mathrm{~m}^{-3}$, $n_{e 0}=4 \times 10^{13} \mathrm{~m}^{-3}$ and in (b) we have $k_{B} T_{i}=0.3 \mathrm{eV}, k_{B} T_{e}=8 \mathrm{eV}$.

As described in Sec. II D, the charge of the dust grain is dependent on the temperatures and densities of the Maxwellian species. To determine how the coupling parameter varies with these quantities, the dust charge number, $Z_{d}$, should first be calculated according to Eq. (20). Figure 1 shows how the dust charge number, $Z_{d}$, is dependent on the parameters used in this investigation, and it is seen to vary between approximately 1100 and 3200 for this parameter range. Using Eqs. (1) and (56), it then can be shown that this corresponds to values of $\Gamma$ which fall approximately in the desired range.

The lower bound for the velocity of a solitary wave is given by the sound speed in the plasma, and so it is important to consider the linear case, even when investigating nonlinear waves. Restoring dimensions to Eq. (39) we see that the dispersion relation may be written as

$$
\omega^{2}=\frac{k_{B} T_{0}}{m_{d}}\left(\frac{\alpha k^{2}}{1+\lambda_{D 0}^{2} k^{2}}+\beta k^{2}\right),
$$

which, in the limit $k \lambda_{D 0} \ll 1$, shows a predicted phase velocity of

$$
v_{\mathrm{ph}}=\frac{\omega}{k}=\sqrt{\frac{k_{B}\left(T_{0}+T_{\star 0}+T_{\star 11}-T_{\star 12}\right)}{m_{d}}} .
$$

In this and the following equations, the terms written $T_{\star i j}$ are the electrostatic temperature perturbation coefficients in dimensional form, corresponding to Eqs. (30)-(34). Figure 2 shows how the phase velocity varies with the parameters under investigation.
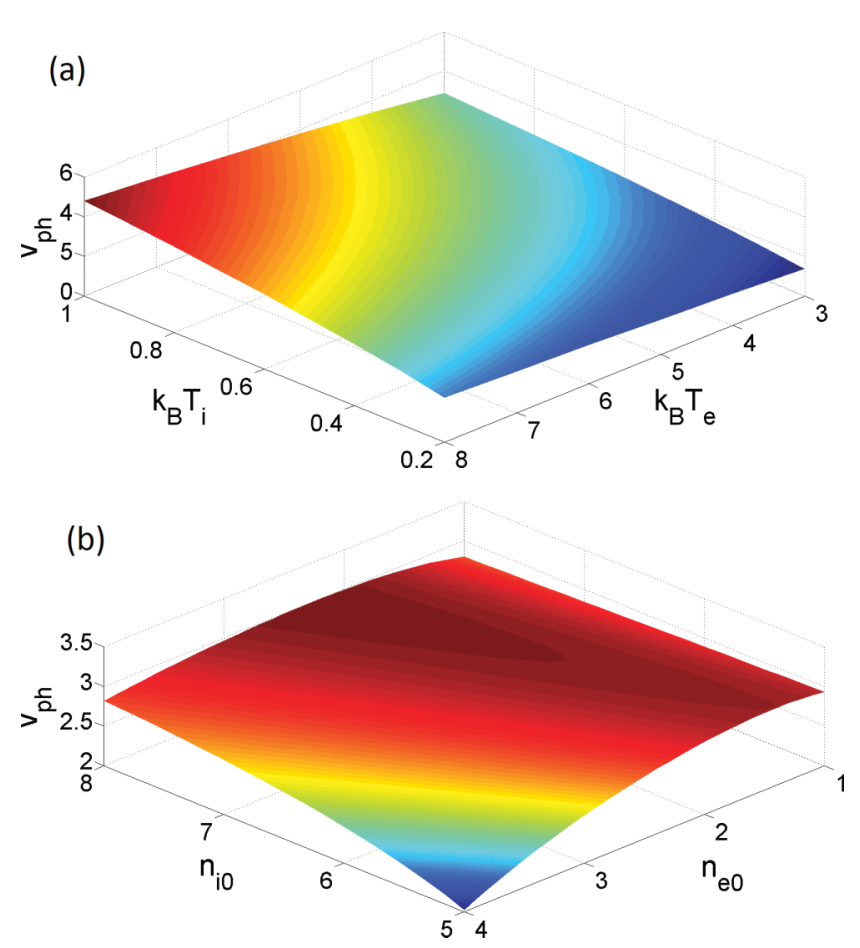

FIG. 2. (Color online) The phase velocity, $v_{\mathrm{ph}}(\mathrm{cm} / \mathrm{s})$ of the dustacoustic waves studied in this investigation, as functions of (a) the temperatures $(\mathrm{eV})$ and $(\mathrm{b})$ the densities (in units of $10^{13} \mathrm{~m}^{-3}$ ) of the Maxwellian species. In both plots we have $m_{d}=1 \times 10^{-13} \mathrm{~kg}, r_{d}=$ $0.2 \mu \mathrm{m}$, and $\sigma=1.3732 \times 10^{-5}$. In (a) we have $n_{i 0}=7 \times 10^{13} \mathrm{~m}^{-3}$, $n_{e 0}=4 \times 10^{13} \mathrm{~m}^{-3}$ and in (b) we have $k_{B} T_{i}=0.3 \mathrm{eV}, k_{B} T_{e}=8 \mathrm{eV}$.

Restoring dimensions to Eq. (49) and rearranging, we obtain

$$
\frac{\partial \Phi_{1}}{\partial \tau}+A \Phi_{1} \frac{\partial \Phi_{1}}{\partial \xi}+B \frac{\partial^{3} \Phi_{1}}{\partial \xi^{3}}=0,
$$

in which we have

$$
A=\frac{Z_{d} e}{\left(m_{d} k_{B} T_{0}\right)^{1 / 2}} \tilde{A}
$$

and

$$
B=\frac{\epsilon_{0}\left(k_{B} T_{0}\right)^{3 / 2}}{Z_{d}^{2} e^{2} n_{d 0} m_{d}^{1 / 2}} \tilde{B} .
$$

This gives a solution in the laboratory frame of

$$
\Phi(x, t)=\Phi_{m} \operatorname{sech}^{2}\left(\frac{\chi}{\Delta}\right),
$$

which is the dimensional form of Eq. (54). Since $\left|\Phi_{m}\right|=$ $3 \delta V /|A|$ and $\Delta=\sqrt{4 B / \delta V}$, we see that the amplitude and width of the solitary waves have two main dependences. The first is a dependence on the velocity of the solitary wave relative to the sound speed in the plasma, $\delta V$, such that the amplitude of the solitary wave is directly proportional, and the width inversely proportional to the square root of its magnitude. This parameter is related to the strength of the perturbation and we see that, for constant $A$ and $B$, faster solitary waves will be taller and thinner than slower ones. We also see the well-known relation that for a particular plasma system, the product $\left|\Phi_{m}\right| \Delta^{2}$ is independent of $\delta V$. 

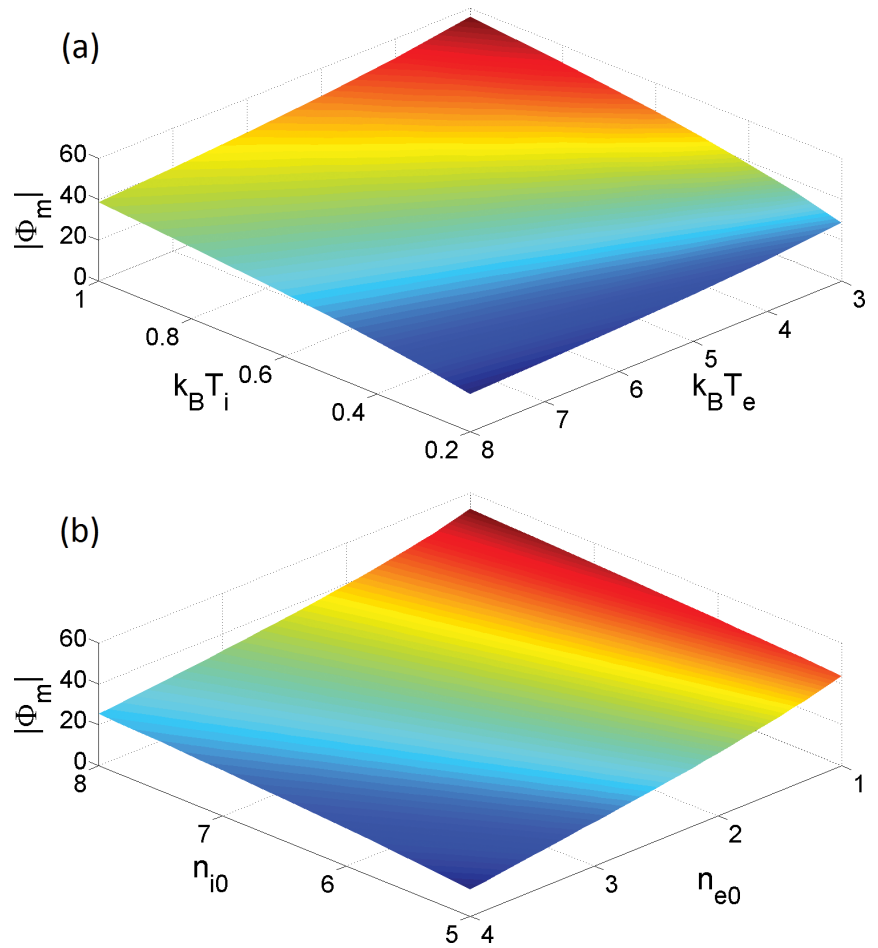

FIG. 3. (Color online) The amplitude, $\left|\Phi_{m}\right|(\mathrm{mV})$ of the potential perturbations associated with the solitary waves studied in this investigation, as functions of (a) the temperatures $(\mathrm{eV})$ and (b) the densities (in units of $10^{13} \mathrm{~m}^{-3}$ ) of the Maxwellian species. In both plots we have $m_{d}=1 \times 10^{-13} \mathrm{~kg}, r_{d}=0.2 \mu \mathrm{m}, \sigma=1.3732 \times 10^{-5}$, and $\delta V=2 \mathrm{~mm} / \mathrm{s}$. In (a) we have $n_{i 0}=7 \times 10^{13} \mathrm{~m}^{-3}, n_{e 0}=$ $4 \times 10^{13} \mathrm{~m}^{-3}$ and in (b) we have $k_{B} T_{i}=0.3 \mathrm{eV}, k_{B} T_{e}=8 \mathrm{eV}$.

The second dependence is on the coefficients of the KdV equation, $A$ and $B$, which are unique to the model being presented. By holding $\delta V$ constant, we demonstrate, in Figs. 3 and 4 , the effects that the variation of these coefficients with equilibrium plasma parameters have on the amplitude and width of solitary waves. We emphasize that, although we have held $\delta V$ constant, in the laboratory frame the speeds of the solitary waves differ, due to the sound speed being dependent on these parameters. By noting the range of phase velocities shown in Fig. 2, we see that Figs. 3 and 4 correspond to solitary waves traveling with a Mach number range of $M=V / v_{\mathrm{ph}}=1.04-1.15$.

\section{THE EFFECTS OF STRONG COUPLING BETWEEN DUST PARTICLES ON DUST-ACOUSTIC WAVES}

In this section, we present a discussion on how strong coupling between the dust particles, described via the electrostatic temperature model, affects the attributes of the linear and nonlinear dust-acoustic waves. We do this by comparing the model presented in this paper, which in this section we denote as the $T_{\star} \equiv T_{\star}\left(n_{d}, \Phi\right)$ case, with a model in which we set the electrostatic temperature terms to zero such that $T_{\star}=0$. This model is well documented and may be found, for example, in Ref. [1], although using a scaling which differs from that in this article. In addition to this, we also show the effects of the inclusion of the electrostatic temperature perturbation terms by comparison to a model in which the electrostatic
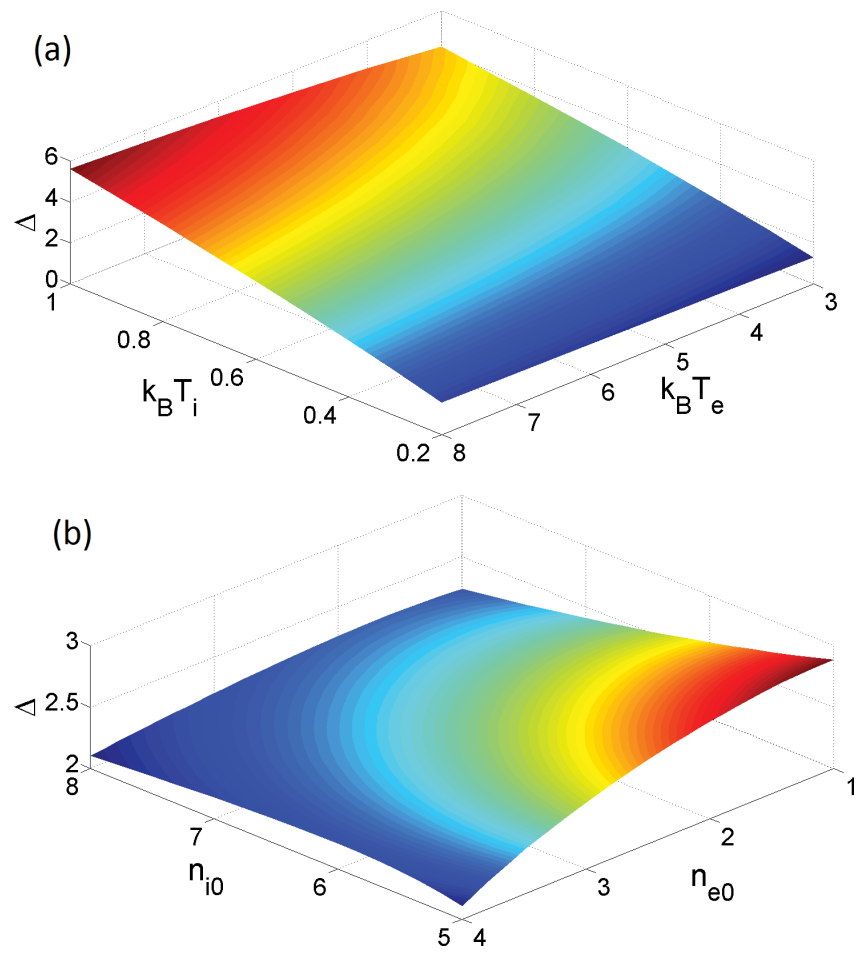

FIG. 4. (Color online) The width, $\Delta$ (mm), of the potential perturbations associated with the solitary waves studied in this investigation, as functions of (a) the temperatures (eV) and (b) the densities (in units of $10^{13} \mathrm{~m}^{-3}$ ) of the Maxwellian species. In both plots we have $m_{d}=1 \times 10^{-13} \mathrm{~kg}, r_{d}=0.2 \mu \mathrm{m}, \sigma=1.3732 \times 10^{-5}$, and $\delta V=2 \mathrm{~mm} / \mathrm{s}$. In (a) we have $n_{i 0}=7 \times 10^{13} \mathrm{~m}^{-3}, n_{e 0}=$ $4 \times 10^{13} \mathrm{~m}^{-3}$ and in (b) we have $k_{B} T_{i}=0.3 \mathrm{eV}, k_{B} T_{e}=8 \mathrm{eV}$.

temperature is set to its equilibrium value such that $T_{\star} \equiv T_{\star 0}$, which may be found, for example, in Ref. [20]. These models may be recovered from the equations presented in this text in the appropriate limits, which are discussed in this section.

\section{A. Linear dust-acoustic waves}

We start with the linear case, and by inspection of the form of Eq. (39), we see that the defining terms for each model are $\alpha$ and $\beta$, the values of which are displayed in Table III. Figure 5 presents the calculated dispersion relations for the three models.

\section{Short-wavelength limit, $k \lambda_{D 0} \gg 1$}

As was demonstrated by Yaroshenko et al. [18], a feature of the dispersion relation which is indicative of strong coupling is a transition into a regime similar to that of the thermal mode at high wave numbers. This indicates that the electrostatic interactions between the dust particles produce an effect

TABLE III. Values of $\alpha$ and $\beta$ for the three models under comparison.

\begin{tabular}{lcc}
\hline \hline Model & $\alpha$ & $\beta$ \\
\hline$T_{\star} \equiv T_{\star}\left(n_{d}, \phi\right)$ & $1-d_{12}$ & $d_{0}+d_{11}$ \\
$T_{\star} \equiv T_{\star 0}$ & 1 & $d_{0}$ \\
$T_{\star}=0$ & 1 & 0 \\
\hline \hline
\end{tabular}




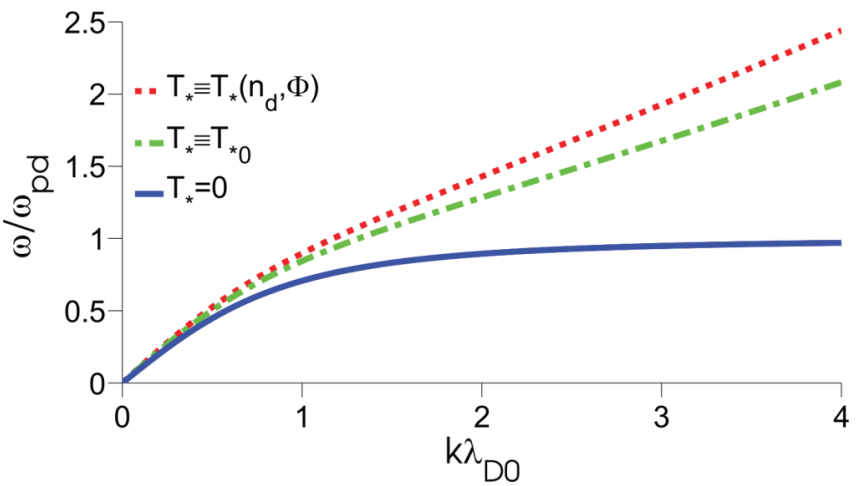

FIG. 5. (Color online) The normalized dispersion relation for three different models of dust-acoustic waves. Here we have $n_{i 0}=7 \times 10^{13} \mathrm{~m}^{-3}, n_{e 0}=4 \times 10^{13} \mathrm{~m}^{-3}, k_{B} T_{i}=0.3 \mathrm{eV}$, $k_{B} T_{e}=8 \mathrm{eV}, \quad m_{d}=1 \times 10^{-13} \mathrm{~kg}, \quad r_{d}=0.2 \mu \mathrm{m}$, and $\sigma=$ $1.3732 \times 10^{-5}$.

analogous to that of a temperature, and we see this effect clearly in Fig. 5. Here, the term responsible for this effect is $\beta$ in Eq. (39). In the weakly coupled case, since we do not consider any temperature-like terms, we have $\beta=0$ and so the wave frequency saturates at the dust plasma frequency for high wave numbers, as can be seen from the solid blue line in Fig. 5. For the strongly coupled models, we have nonzero $\beta$ terms, so for large wave numbers, these models predict a transition to the thermal mode, such that $\tilde{\omega} \rightarrow \sqrt{\beta} \tilde{k}$. Since the value of $\beta$ is larger for the $T_{\star} \equiv T_{\star}\left(n_{d}, \Phi\right)$ model than the $T_{\star} \equiv T_{\star 0}$ case, we see that the inclusion of dust electrostatic temperature perturbations in the model makes this effect even more pronounced.

\section{Long-wavelength limit, $k \lambda_{D 0} \ll 1$}

In the case of low wave numbers such that $k \lambda_{D 0} \ll 1$, Yaroshenko et al. [18] predicted an increase in the phase velocity of the wave due to the effects of strong coupling. Here, we see that, in this limit, $v=\sqrt{\alpha+\beta}$. For the scaling we have chosen, the $T_{\star}=0$ model has a phase velocity of 1 . Since the phase velocity for the $T_{\star} \equiv T_{\star 0}$ model is $\sqrt{1+d_{0}}$, we see that an effect of including strong coupling in the model is an increase in the phase velocity in the longwavelength limit. In this paper, for the $T_{\star} \equiv T_{\star}\left(n_{d}, \Phi\right)$ case, we have a phase velocity of $v=\sqrt{1+d_{0}+d_{11}-d_{12}}$. For the parameters considered in Sec. V, we have $d_{11}>d_{12}$ across the entire range, so we see that the electrostatic temperature perturbations further increase the predicted phase velocity. The difference in phase velocity between the models is a function of the equilibrium plasma parameters. To get a sense of the magnitude that these differences amount to, we produced graphs similar to Fig. 2 for the two other models, which are excluded from this work for brevity. In doing so, it may be seen that the approach used in this paper predicts phase speeds that are up to approximately $20 \%$ greater than the $T_{\star}=0$ model and up to approximately $5 \%$ greater than the $T_{\star} \equiv T_{\star 0}$ model for the parameter range considered in Sec. V.

\section{B. Nonlinear dust-acoustic solitary waves}

We now discuss the nonlinear case. By considering the form of Eq. (55), we see there are two key factors affecting the attributes of the nonlinear solitary waves. The first is the sound speed excess, $\delta \tilde{V}$, of the solitary wave, and the second is the values of the coefficients in the $\mathrm{KdV}$ equation, $\tilde{A}$ and $\tilde{B}$. Both of these factors are dependent on the model used, so we will now discuss each of them in turn.

\section{Differences in the calculated sound speed excess}

For a measured solitary wave speed in the laboratory frame $\tilde{V}$, each model will give a different value of $\delta \tilde{V}$ on which the amplitude and width of the solitary waves are dependent. For example, using the same parameters which were used for the comparison in Fig. 5, the normalized phase speeds, $v$, for the dynamically varying electrostatic temperature model, the constant electrostatic temperature model, and the electrostatically cold model are $1.14,1.10$, and 1 , respectively. For a solitary wave moving in the laboratory frame with a normalized velocity of $\tilde{V}=1.2$, the three models then give $\delta \tilde{V}=(\tilde{V}-v)$ of $0.06,0.1$, and 0.2 , respectively. Since the amplitude is directly proportional to, and the width is inversely proportional to, the square root of $\delta \tilde{V}$, it is seen that this factor contributes to the strongly coupled models predicting shorter but wider solitary waves.

This factor is most important for lower values of $\tilde{V}$. To see this, let us consider two separate models, with phase velocities $v_{1}$ and $v_{2}$, respectively. One may arbitrarily choose $v_{2}>v_{1}$. It then follows that $\delta \tilde{V}_{1}=\tilde{V}-v_{1}>\delta \tilde{V}_{2}$. The ratio $\delta \tilde{V}_{1} / \delta \tilde{V}_{2}$ may then be seen to increase monotonically as $\tilde{V}$ is decreased and approaches $v_{2}$. Thus, it follows that the relative differences in the predicted amplitudes and widths of the solitary waves, arising from the deviations in $\delta \tilde{V}$ between the models, are greatest for lower values of $\tilde{V}$.

\section{Differences in the KdV coefficients}

We now compare the $\tilde{A}$ and $\tilde{B}$ coefficients in the $\mathrm{KdV}$ equation for the three models, which leads to differences in the amplitude and width of the predicted solitary waves.

Accounting for the differences in $\alpha$ and $v=\sqrt{\alpha+\beta}$ between the models, it may be seen that the $\mathrm{KdV}$ coefficients for the $T_{\star}=0$ and $T_{\star} \equiv T_{\star 0}$ models are

$$
\tilde{A}=-\frac{3+2 c_{2}}{2} \quad \tilde{B}=\frac{1}{2}
$$

and

$$
\tilde{A}=-\frac{3+2 c_{2}+2 d_{0}}{2 \sqrt{1+d_{0}}} \quad \tilde{B}=\frac{1}{2 \sqrt{1+d_{0}}},
$$

respectively. Having calculated the values of these coefficients across the parameter range described in Sec. V, we find that the $T_{\star} \equiv T_{\star}\left(n_{d}, \Phi\right)$ model presented in this paper predicts values of $\tilde{A}$ which are greater in magnitude than those predicted by the $T_{\star} \equiv T_{\star 0}$ and $T_{\star}=0$ models by up to a maximum of approximately $8 \%$ and $15 \%$, respectively. We find that the corresponding decrease in $\tilde{B}$ reaches approximately $3 \%$ and $8 \%$, respectively. The percentage difference in the coefficients predicted by each model varies across the equilibrium parameter range, so we just give the maximum values to give a sense of the magnitude of these changes. The increase in the absolute value of the $\tilde{A}$ coefficient contributes to the prediction of lower amplitude solitary waves for the strongly coupled models. The decrease in $\tilde{B}$ contributes to a narrowing of the 


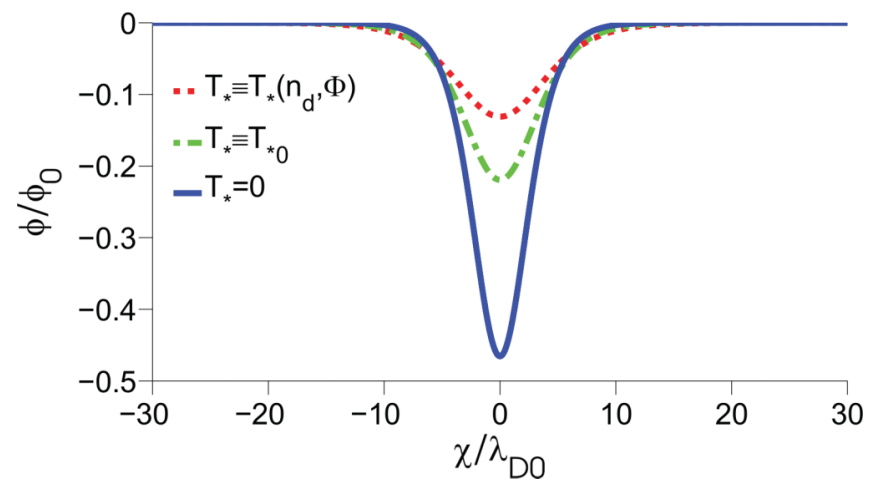

FIG. 6. (Color online) Normalized solitary wave structures for three different models. Here we have $n_{i 0}=7 \times 10^{13} \mathrm{~m}^{-3}, n_{e 0}=4 \times$ $10^{13} \mathrm{~m}^{-3}, k_{B} T_{i}=0.3 \mathrm{eV}, k_{B} T_{e}=8 \mathrm{eV}, m_{d}=1 \times 10^{-13} \mathrm{~kg}, r_{d}=$ $0.2 \mu \mathrm{m}, \sigma=1.3732 \times 10^{-5}$, and $\tilde{V}=1.2$.

solitary waves for the strongly coupled models, which is in contrast to the effect arising from the reduction of the value of $\delta \tilde{V}$, which leads to a widening. The net effect on the predicted width of the solitary waves thus depends on which factor is dominant, which is determined by the specific equilibrium plasma parameters and the solitary wave velocity, $\tilde{V}$.

Figure 6 shows solitary waves predicted by the three models for typical plasma parameters. Here we see that the combination of the reduction of $\delta \tilde{V}$ and the increase of $|\tilde{A}|$ results in a large decrease in the amplitude of the solitary waves for the strongly coupled models. The effect of strong coupling on the width is more subtle, but Fig. 6 shows slightly wider solitary waves for the strongly coupled models, which indicates that for these parameters the effect of the reduction in $\delta \tilde{V}$ dominates over the effect of the reduction in $\tilde{B}$.

\section{CONCLUSIONS}

In this paper we have theoretically investigated both linear and nonlinear dust-acoustic waves in a dusty plasma in which the dust particles are strongly coupled. We have modelled the effects of strong coupling by utilizing the electrostatic temperature approach of Yaroshenko et al. [18]. Uniquely, we have considered perturbations in the electrostatic temperature, which is a function of the dust number density and electrostatic potential, in the locality of the wave, and then demonstrated how these affect the linear dispersion relation and the Korteweg-de Vries equation.

Our main objectives were to investigate how the dynamics of the waves change for a range of equilibrium plasma parameters, to describe what effects strong coupling has on the waves, and to provide an overview of how electrostatic temperature perturbations affect the model. In summary, the main conclusions of our paper are as follows:

(1) The phase velocity of the dust-acoustic wave, as well as the amplitude and width of solitary waves, are seen to vary significantly with the equilibrium plasma parameters. This is shown in Figs. 2, 3, and 4, respectively, where we have displayed how the variation of densities and temperatures of the Maxwellian species affect these attributes.
(2) The predicted phase velocity, $v$, is seen to be larger when strong coupling effects are accommodated in the model via the electrostatic temperature approach.

(3) The amplitude of the predicted nonlinear solitary waves is seen to be reduced when strong coupling is included in the model, due to an increase in the absolute value of the nonlinear coefficient, $\tilde{A}$, in the $\mathrm{KdV}$ equation. In addition to this, if we consider a solitary wave traveling with a set velocity in the laboratory frame $\tilde{V}$, the increase in phase velocity arising from strong coupling effects will result in a reduction in the predicted sound speed excess, $\delta \tilde{V}=\tilde{V}-v$, compared to the weakly coupled model. This will then result in a further reduction in the amplitude predicted by the strongly coupled models.

(4) The width of the predicted nonlinear solitary waves may be seen to either increase or decrease with the inclusion of strong coupling effects, depending on which of two factors is dominant. The first, contributing to a narrowing of the solitary waves for the parameters under investigation, is the reduction of the $\tilde{B}$ coefficient in the $\mathrm{KdV}$ equation. The second factor, contributing to a widening of the solitary waves, is the reduction in $\delta \tilde{V}$ brought about by the increased phase velocity in the strongly coupled model. Which factor dominates is determined by the specific equilibrium plasma parameters and the solitary wave velocity, $\tilde{V}$.

(5) The inclusion of the electrostatic temperature perturbations in the model is seen to amplify the effects of strong coupling mentioned in points (2)-(4).

We have seen that the difference between the inclusion or exclusion of the perturbations of the electrostatic temperature affects the value of the linear phase velocity by only a small amount (the magnitude of this difference depends on the specific equilibrium plasma conditions but is seen to be at most $\sim 5 \%$ for the parameter range under investigation). For solitary waves, however, since the sound speed excess will be changed, this seemingly small modification can result in large differences in the amplitude and width between the two models when combined with the changes in the $\mathrm{KdV}$ coefficients. We show, therefore, that the consideration of the dynamic variation of the electrostatic temperature can provide important modifications to more simple models.

The dust charge number $Z_{d}$, which in this case was derived using an orbit motion limited approach, is found to vary significantly with the equilibrium plasma parameters and this has a substantial effect on the dynamics of the wave modes. For a more complete understanding of the system, perturbations of the dust charge number may also show important additions to the model, since $Z_{d}$ is actually dependent on the dynamically varying electron and ion densities.

\section{ACKNOWLEDGMENTS}

I.K. warmly acknowledges support from UK Engineering and Physical Sciences Research Council (EPSRC) via Grant No. EP/I031766/1. The work of I.K. and S.S. was supported by a UK EPSRC Science and Innovation award to the Centre for Plasma Physics, Queen's University Belfast (Grant No. EP/D06337X/1). The work of M.A.H. is supported in part by the National Research Foundation of South Africa (NRF). 


\section{APPENDIX A: THE CALCULATION OF THE DUST CHARGE NUMBER VIA THE LAMBERT $W$ FUNCTION}

According to OML theory, the electron and ion currents onto the dust particle may be written as

$$
\begin{aligned}
& I_{e}=I_{e 0} \exp \left(\frac{e \Phi_{d}}{k_{B} T_{e}}\right), \\
& I_{i}=I_{i 0}\left(1-\frac{e \Phi_{d}}{k_{B} T_{i}}\right),
\end{aligned}
$$

with

$$
I_{s 0}=\sqrt{8 \pi} r_{d}^{2} n_{s 0} q_{s} \sqrt{\frac{k_{B} T_{s}}{m_{s}}}
$$

for species $s=e, i$. Here $\Phi_{d}$ is the difference between the grain and plasma potentials. Setting $I_{i}+I_{e}=0$ gives

$$
-\mu \sqrt{\frac{1}{\sigma \theta}} \exp \left(\frac{e \Phi_{d}}{k_{B} T_{e}}\right)+\left(1-\frac{e \Phi_{d}}{k_{B} T_{i}}\right)=0,
$$

where we have defined $\sigma=m_{e} / m_{i}, \mu=n_{e 0} / n_{i 0}, \theta=T_{i} / T_{e}$. Multiplying through by $\theta \exp \left(\theta-e \Phi_{d} / k_{B} T_{e}\right)$ and rearranging then gives

$$
\left(\theta-\frac{e \Phi_{d}}{k_{B} T_{e}}\right) \exp \left(\theta-\frac{e \Phi_{d}}{k_{B} T_{e}}\right)=\mu \sqrt{\frac{\theta}{\sigma}} \exp (\theta) .
$$

This equation is of the form $X \exp (X)=f(\mu, \theta)$, the solution to which may be written as $X=W(f(\mu, \theta))$, where $W$ denotes the Lambert $W$ function. This equation thus may be expressed as

$$
\theta-\frac{e \Phi_{d}}{k_{B} T_{e}}=W\left[\mu \sqrt{\frac{\theta}{\sigma}} \exp (\theta)\right] .
$$

By then substituting $\Phi_{d}=-Z_{d} e /\left(4 \pi \epsilon_{0} r_{d}\right)$ into this equation and rearranging, we obtain our expression for the dust charge number, Eq. (20).

\section{APPENDIX B: DERIVATION OF ELECTROSTATIC TEMPERATURE COEFFICIENTS}

In this Appendix, we outline a derivation of the electrostatic temperature coefficients which were presented in Sec. III. The electrostatic temperature depends on the local screening length in the plasma via the lattice parameter $\kappa$, and we use this as the starting point. Looking at the normalized Debye length, we see that

$$
\begin{aligned}
\frac{\lambda_{D}}{\lambda_{D 0}} & =\left(\frac{n_{i 0} T_{e}+n_{e 0} T_{i}}{n_{i} T_{e}+n_{e} T_{i}}\right)^{1 / 2} \\
& =\left[\frac{1+\mu \theta}{\exp \left(-\frac{e \Phi}{k_{B} T_{i}}\right)+\mu \theta \exp \left(\frac{e \Phi}{k_{B} T_{e}}\right)}\right]^{1 / 2} .
\end{aligned}
$$

We may write the electrostatic potential as $\Phi=$ $\phi \Phi_{0}$, where the normalization quantity $\Phi_{0}=k_{B} T_{0} / e=$ $[(1-\mu) /(1+\mu \theta)]\left(k_{B} T_{i} / e\right)$. The denominator of the last term in Eq. (B1) may then be written as

$$
\exp \left[-\frac{(1-\mu)}{(1+\mu \theta)} \phi\right]+\mu \theta \exp \left[\frac{(1-\mu) \theta}{(1+\mu \theta)} \phi\right] .
$$

By expressing $\phi$ in a power series of $\epsilon$ and Taylor expanding, Eq. (B1) then simplifies to

$$
\frac{\lambda_{D}}{\lambda_{D 0}}=\left[\frac{1}{\sum_{j=0}^{\infty}(j+1) c_{j+1} \phi^{j}}\right]^{1 / 2},
$$

where $c_{j}$ refers to the $j^{\text {th }}$ coefficient in Poisson's equation, as defined in Sec. II C.

To derive the KdV equation we only require up to the second order, so we take the lattice parameter, $\kappa$, to be

$$
\kappa=\frac{1}{\lambda_{D} n_{d}^{1 / 3}}=\kappa_{0}\left(1+2 c_{2} \phi+3 c_{3} \phi^{2}\right)^{1 / 2} n^{-1 / 3} .
$$

By expressing the dynamic variables in series of $\epsilon$ and Taylor expanding, we arrive at an equation for the perturbations in the lattice parameter, such that

$$
\kappa=\kappa_{0}+\epsilon \kappa_{1}+\epsilon^{2} \kappa_{2}
$$

with

$$
\kappa_{1}=-\frac{1}{3} n_{1}+c_{2} \phi_{1}
$$

and

$$
\kappa_{2}=-\frac{1}{3} n_{2}+c_{2} \phi_{2}+\frac{2}{9} n_{1}^{2}-\frac{c_{2}}{3} n_{1} \phi_{1}+\frac{\left(3 c_{3}-c_{2}^{2}\right)}{2} \phi_{1}^{2} .
$$

This equation for $\kappa$ can now be substituted into Eq. (5) to obtain the electrostatic temperature perturbation terms. The calculation can be divided up into three main components, with

$$
\begin{aligned}
n_{d}^{1 / 3} & \simeq n_{d 0}^{1 / 3}\left(1+\epsilon n_{1}+\epsilon^{2} n_{2}\right)^{1 / 3} \\
& \simeq n_{d 0}^{1 / 3}\left[1+\epsilon \frac{n_{1}}{3}+\epsilon^{2} \frac{1}{9}\left(3 n_{2}-n_{1}^{2}\right)\right], \\
(1+\kappa) & \simeq\left(1+\kappa_{0}\right)\left(1+\epsilon \frac{\kappa_{1}}{1+\kappa_{0}}+\epsilon^{2} \frac{\kappa_{2}}{1+\kappa_{0}}\right),
\end{aligned}
$$

and

$$
\begin{aligned}
\exp (-\kappa) & \simeq \exp \left(-\kappa_{0}-\epsilon \kappa_{1}-\epsilon^{2} \kappa^{2}\right) \\
& =\exp \left(-\kappa_{0}\right) \exp \left(-\epsilon \kappa_{1}-\epsilon^{2} \kappa_{2}\right) \\
& \simeq \exp \left(-\kappa_{0}\right)\left[1-\epsilon \kappa_{1}+\epsilon^{2} \frac{\left(\kappa_{1}^{2}-2 \kappa_{2}\right)}{2}\right] .
\end{aligned}
$$

The product of these three equations, along with the constants appearing at the beginning of Eq. (5), gives, after normalization by $T_{0}$, the electrostatic temperature perturbations that are presented in Sec. III.
[1] P. K. Shukla and A. A. Mamun, Introduction to Dusty Plasma Physics (Institute of Physics, Bristol, 2002).

[2] G. E. Morfill, H. M. Thomas, B. M. Annaratone, A. V. Ivlev, R. A. Quinn, A. P. Nefedov, and V. E. Fortov, AIP Conf. Proc. 649, 91 (2002).
[3] N. N. Rao, P. K. Shukla, and M. Y. Yu, Planet. Space Sci. 38, 543 (1990).

[4] A. Barkan, R. L. Merlino, and N. D’Angelo, Phys. Plasmas 2, 3563 (1995).

[5] H. Ikezi, Phys. Fluids 29, 1764 (1986). 
[6] J. H. Chu, and I. Lin, Phys. Rev. Lett. 72, 4009 (1994).

[7] H. Thomas, G. E. Morfill, V. Demmel, J. Goree, B. Feuerbacher, and D. Möhlmann, Phys. Rev. Lett. 73, 652 (1994).

[8] Y. Hayashi and K. Tachibana, Jpn. J. Appl. Phys. 33, L804 (1994).

[9] G. E. Morfill, H. M. Thomas, U. Konopka, H. Rothermel, M. Zuzic, A. Ivlev, and J. Goree, Phys. Rev. Lett. 83, 1598 (1999).

[10] X. Wang and A. Bhattacharjee, Phys. Plasmas 4, 3759 (1997).

[11] M. Rosenberg and G. Kalman, Phys. Rev. E 56, 7166 (1997).

[12] M. S. Murillo, Phys. Plasmas 5, 3116 (1998).

[13] P. K. Kaw, Phys. Plasmas 8, 1870 (2001).

[14] G. Gozadinos, A. V. Ivlev, and J. P. Boeuf, New J. Phys. 5, 32 (2003).

[15] V. V. Yaroshenko, F. Verheest, H. M. Thomas, and G. E. Morfill, New J. Phys. 11, 073013 (2009).

[16] V. V. Yaroshenko, V. Nosenko, M. A. Hellberg, F. Verheest, H. M. Thomas, and G. E. Morfill, New J. Phys. 12, 073038 (2010).
[17] V. V. Yaroshenko, M. H. Thoma, H. M. Thomas, and G. E. Morfill, IEEE Trans. Plasma Sci. 38, 869 (2010).

[18] V. V. Yaroshenko, V. Nosenko, and G. E. Morfill, Phys. Plasmas 17, 103709 (2010).

[19] A. A. Mamun and P. K. Shukla, Europhys. Lett. 87, 55001 (2009).

[20] A. A. Mamun, K. S. Ashrafi, and P. K. Shukla, Phys. Rev. E 82, 026405 (2010).

[21] V. Nosenko, S. K. Zhdanov, S.-H. Kim, J. Heinrich, R. L. Merlino, and G. E. Morfill, Europhys. Lett. 88, 65001 (2009).

[22] P. Bandyopadhyay, G. Prasad, A. Sen, and P. K. Kaw, Phys. Rev. Lett. 101, 065006 (2008).

[23] J. Goree, Plasma Sources Sci. Technol. 3, 400 (1994).

[24] X. Wang and A. Bhattacharjee, Phys. Plasmas 3, 1189 (1996).

[25] A. E. Dubinov and I. D. Dubinova, J. Plasma Phys. 71, 715 (2005).

[26] H. Washimi and T. Taniuti, Phys. Rev. Lett. 17, 996 (1966).

[27] O. S. Vaulina and S. A. Khrapak, J. Theor. Exp. Phys. 90, 287 (2000). 